[Article]

www.whxb.pku.edu.cn

\title{
原位椭圆偏振光谱研究多孔阳极氧化铝初期生长过程
}

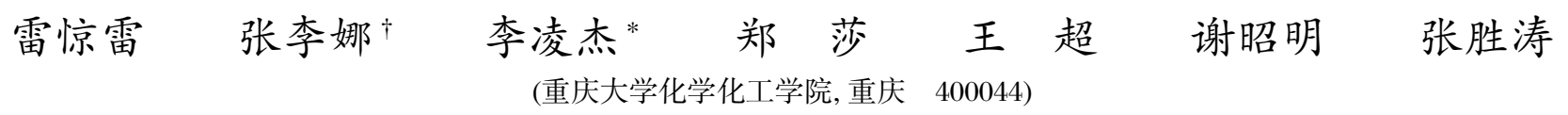

\begin{abstract}
摘要： 根据铝阳极氧化初期不同阶段 $\mathrm{Al}-\mathrm{H}_{2} \mathrm{SO}_{4}$ 体系的界面特征建立多个光学模型, 并采用有效介质近似解析 了高时间分辨率的原位㮁圆偏振光谱数据, 详细获得铝阳极氧化初期的 $\mathrm{Al}_{2} \mathrm{O}_{3}-\mathrm{Al}$ 界面层、 $\mathrm{Al}_{2} \mathrm{O}_{3}$ 阻挡层以及多孔 层组成、厚度变化的动态信息. 根据光学模型及计算结果, 可以清楚地分辨铝阳极氧化过程中的阻挡层形成、孔 洞萌生、孔洞发展以及多孔层稳定生长等 4 个阶段. 在多孔层稳定生长阶段, 阻挡层厚度及多孔层孔隙率几乎不 变, 而多孔层厚度随时间线性增长, 其速率为 $5.8 \mathrm{~nm} \cdot \mathrm{s}^{-1}$.
\end{abstract}

关键词： 铝；阳极氧化；模板；椭圆偏振光谱；有效介质近似

中图分类号: O646.54

\section{In situ Ellipsometric Study on the Initial Stages of Porous Anodization of Aluminum}

\author{
LEI Jing-Lei ZHANG Li-Na $\quad$ LI Ling-Jie* ${ }^{*} \quad$ ZHENG Sha \\ WANG Chao XIE Zhao-Ming ZHANG Sheng-Tao \\ (College of Chemistry and Chemical Engineering, Chongqing University, Chongqing 400044, P. R. China)
}

\begin{abstract}
The initial stages of the anodization of aluminum are influential during the preparation of anodic aluminum oxide nanotemplates and provide an insight into their formation mechanism. The formation and development of both the barrier layer and the porous layer are involved. In this paper, in situ ellipsometric spectra with a high time-resolution were collected. To deconvolute the ellipsometric spectra, several optical models were extracted from the physical models of the $\mathrm{Al}-\mathrm{H}_{2} \mathrm{SO}_{4}$ interface. Using these models and the effective medium approximation, detailed information about the composition and thickness of the $\mathrm{Al}_{2} \mathrm{O}_{3}$ - $\mathrm{Al}$ interphase, $\mathrm{Al}_{2} \mathrm{O}_{3}$ barrier layer, and porous layer was acquired. Based on the deconvoluted results and the optical models, 4 stages of the porous anodization of aluminum, i.e., the barrier layer grows, pores form, pores enlarge, and pores grow at a stable rate, were clearly distinguished. Moreover, during the last stage the thickness of the porous layer changes linearly with time at a rate of $5.8 \mathrm{~nm} \cdot \mathrm{s}^{-1}$ while both the thickness of the barrier layer and the porosity of the porous layer change very little.
\end{abstract}

Key Words: Aluminum; Anodization; Template; Spectroscopic ellipsometry; Effective medium approximation

多孔阳极氧化铝膜由于具有整齐规则的纳米

的重要模板, 近年来随着纳米技术的迅速发展而受 结构, 是制备低维光学、电学或磁学纳米功能材料

到广泛关注 ${ }^{[1]}$. 然而, 目前的相关研究大多集中于多

Received: February 19, 2010; Revised: April 18, 2010; Published on Web: July 7, 2010.

"Corresponding author. Email: LJLi@cqu.edu.cn.

The project was supported by the National Natural Science Foundation of China (20603049, 20803097), Scientific Research Foundation for the Returned Overseas Chinese Scholars, Ministry of Education, China ([2007]1108-4) and Natural Science Foundation Project of CQ CSTC

(2008BB4174).

国家自然科学基金(20603049, 20803097)和教育部留学回国人员科研启动基金(教外司留[2007]1108-4), 重庆市自然科学基金

(CSTS2008BB4174)资助项目

†现在地址: 四川天华股份有限公司, 邮编 646207.

(C) Editorial office of Acta Physico-Chimica Sinica 
孔阳极氧化铝膜的制备及应用 ${ }^{[2-10]}$, 探讨其多孔结构 的生长机制及生长动力学的理论研究相对较少 ${ }^{[11-12]}$. 这一方面是由于铝阳极氧化过程涉及复杂的化学、 电学、力学等多种因素的耦合而导致理论研究困难, 另一方面也是由于铝化学性质活泼而致使很难应用 常规研究方法获取反应中间过程动态信息并推测其 反应机理.

椭圆偏振光谱法作为一种高灵敏度、非破坏性 的原位光谱电化学研究方法, 可在进行电化学研究 的同时获取电极表面膜层厚度、组成等信息, 为推测 反应机理提供有力证据 ${ }^{[3-14]}$. 将椭圆偏振光谱法应 用于铝阳极氧化过程的研究已有少量报道 ${ }^{[15-18]}$, 然 而这些研究均是单波长测量或非原位测量, 且时间 分辨率较低, 不能准确、及时地提供反应过程中, 特 别是铝阳极氧化初期的电极表面膜层的实时动态变 化信息. 由于铝阳极氧化初期涉及阻挡层和多孔层 的生成、发展等阶段 ${ }^{[19-20]}$, 对于探明铝多孔阳极氧化 膜的生长机制非常重要, 已引起人们的关注 ${ }^{[2]}$. 随着 仪器制造技术和计算机技术的发展, 单次采集椭圆 偏振光谱数据时间缩短到数十毫秒, 时间分辨率大 大提高 ${ }^{[22]}$. 本文通过解析多孔阳极氧化铝膜生长初 期的高时间分辨率原位椭圆偏振光谱数据, 获取膜 层厚度、组成随时间的变化信息.

\section{1 实 验}

实验采用两电极体系, 工作电极为环氧树脂封 装的纯度为 $99.9 \%$ 的金属 $\mathrm{Al}$ (西南铝业集团有限公 司), 用 $400 \# 、 800 \# 、 1200 \#$ 和 2000 \# 水砂纸逐级打磨 至镜面光滑后进行电化学抛光(在体积比为 $1: 4$ 的 $\mathrm{HClO}_{4}$ 与 $\mathrm{C}_{2} \mathrm{H}_{5} \mathrm{OH}$ 溶液中以 $15.0 \mathrm{~V}$ 电压抛光 1 $\mathrm{min}$ ); 对电极为大 $\mathrm{Pt}$ 片; 电解液为 $1.1 \mathrm{~mol} \cdot \mathrm{L}^{-1}$ 的 $\mathrm{H}_{2} \mathrm{SO}_{4}$ (重庆川东化工有限公司化学试剂厂) 溶液; 采 用直流稳压电源(北京大华电子集团 DH1716A-13) 控制电压为 $16.0 \mathrm{~V}$; 实验温度为 $23.0{ }^{\circ} \mathrm{C}$. 粗圆偏振 光谱(美国 J. A. Woollam 公司 M2000U)测量的波长 $\lambda$ 范围为 600-800 nm, 人射角为 $70^{\circ}$; 仪器单次采集 光谱时间需 $38 \mathrm{~ms}$, 为提高信噪比, 以 40 次采集的 平均值作为输出信号, 即光谱的实际时间分辨率为 $1.5 \mathrm{~s}$ (在实验总时间 $90 \mathrm{~s}$ 内, 共记录 63 次光谱数据); 椭圆偏振数据解析采用仪器自带软件 WVASE 32 . $\mathrm{Al}_{2} \mathrm{O}_{3}$ 的光学常数采用软件提供的数据库 ${ }^{[23]}$; 在进行 阳极氧化之前, 采用椭圆偏振光谱法在空气中测量 并拟合 $\mathrm{Al}$ 基底的光学常数.

\section{2 结果与讨论}

图 1 是氧化电流随时间的变化曲线, 其形状和 文献报道 ${ }^{[20,24]}$ 的非常相似, 表现出先急剧减小, 越过 最低点后逐渐回升直至几乎稳定的特征. 其中插图 为前 $28 \mathrm{~s}$ 的电流-时间曲线放大图, 可以发现约在 $2.6 \mathrm{~s}$ 时, 电流出现最小值.

记录电流的同时记录椭圆偏振光谱数据. 图 2 分别绘出波长为 600 和 $800 \mathrm{~nm}$ 的椭圆偏振光谱参 量 $\Delta-\psi$ 实验曲线. 借鉴解析椭圆偏振光谱的“环境膜层-基底” 经典模型 ${ }^{[13]}$, 可以建立如图 3 所示的 “ $\mathrm{H}_{2} \mathrm{SO}_{4}$ 溶液- $\mathrm{Al}_{2} \mathrm{O}_{3}$ 层- $\mathrm{Al}$ 基底” 3 层膜光学模型. 由 于 $\mathrm{Al}_{2} \mathrm{O}_{3}$ 和 $\mathrm{H}_{2} \mathrm{SO}_{4}$ 的消光系数均为 0 , 根据此模型计 算, 随 $\mathrm{Al}_{2} \mathrm{O}_{3}$ 层厚度增加, $\Delta-\psi$ 理论曲线应为一条封

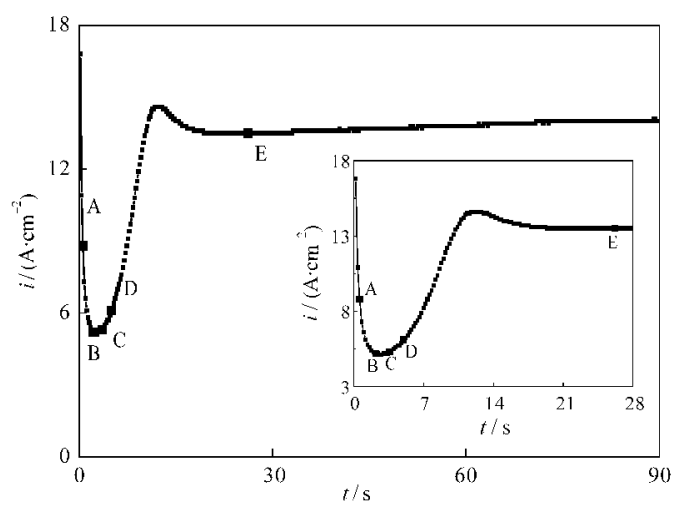

图 $1 \mathrm{Al}$ 在 $1.1 \mathrm{~mol} \cdot \mathrm{L}^{-1} \mathrm{H}_{2} \mathrm{SO}_{4}$ 溶液中的阳极氧化 电流-时间曲线

Fig.1 Anodic current transient of Al in $1.1 \mathrm{~mol} \cdot \mathrm{L}^{-1}$ $\mathrm{H}_{2} \mathrm{SO}_{4}$ solution

The inset shows the current from 0 to $28 \mathrm{~s}$. The letters A, B, C, and D correspond to the time of the first 4 ellipsometric measurements, i.e., $0.6,2.2,3.5,5.0 \mathrm{~s}$, respectively. The letter E corresponds to the time of porous layer starts to grow at a stable rate $(26.2 \mathrm{~s})$, which is determined from the ellipsometric measurements.

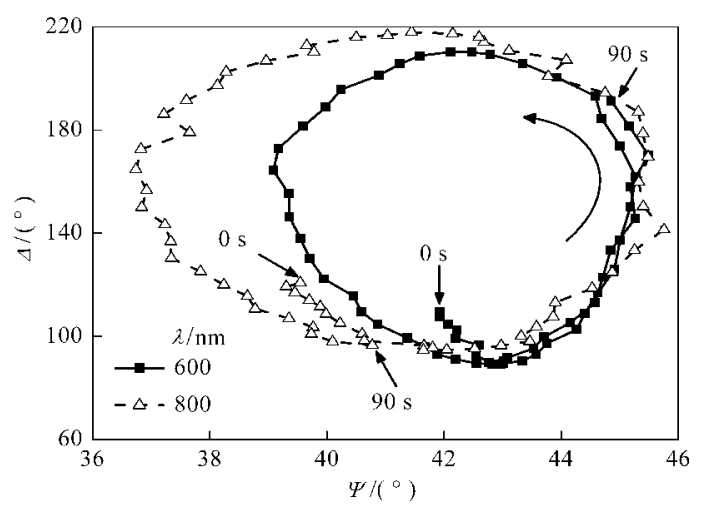

图 2 波长 $\lambda$ 分别为 600 和 $800 \mathrm{~nm}$ 的椭圆偏振光谱 参量 $\Delta-\psi$ 实验曲线

Fig.2 Experimental curves of $\Delta-\psi$ at the wavelength of 600 and $800 \mathrm{~nm}$ 

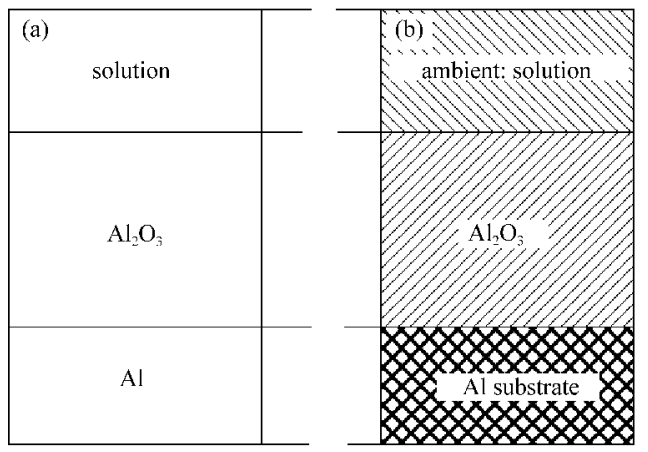

图 3 解析阻挡层形成阶段的椭圆偏振光谱实验数据的 “ $\mathrm{H}_{2} \mathrm{SO}_{4}$ 溶液- $\mathrm{Al}_{2} \mathrm{O}_{3}$ 层- $\mathrm{Al}$ 基底”3 层膜模型

Fig.3 Three-layer model of " $\mathrm{H}_{2} \mathrm{SO}_{4}$ solution- $\mathrm{Al}_{2} \mathrm{O}_{3}$-Al substrate" used to fit the ellipsometric data

(a) physical model; (b) optical model

闭曲线 ${ }^{[25-26 .}$. 但从图 2 可以看出, $\Delta-\psi$ 实验曲线并不 封闭, 表明进行实验的 $90 \mathrm{~s}$ 内, 多孔层已经生成并 显著影响椭圆偏振光谱测量结果. 逐一对各时刻的 $\psi-\lambda$ 和 $\Delta-\lambda$ 曲线进行拟合, 可以得知膜层厚度、组 成随时间的变化信息.

采用图 3 所示的 3 层膜光学模型(仅拟合 $\mathrm{Al}_{2} \mathrm{O}_{3}$ 层厚度)可以很好地拟合对应于通电后 $0.6 \mathrm{~s}$ (图 1 中 标记为 $\mathrm{A}$ 点)的第 1 次原位椭圆偏振光谱数据, 拟 合得到的理论曲线与实验曲线符合很好(如图 4 所 示). 此时, 在 $\mathrm{Al}$ 阳极氧化初期, 将按照如下反应生 成 $\mathrm{Al}_{2} \mathrm{O}_{3}{ }^{[20]}$ :

$2 \mathrm{Al}+3 \mathrm{H}_{2} \mathrm{O} \rightarrow \mathrm{Al}_{2} \mathrm{O}_{3}+6 \mathrm{H}^{+}+6 \mathrm{e}$

$\mathrm{Al}_{2} \mathrm{O}_{3}$ 形成致密的阻挡层, 由于其电阻很大, 随 阻挡层厚度增加, 通过体系的电流迅速减小, 此即文 献中通常认为的 “阻挡层形成”阶段 ${ }^{[19-20]}$. 采用同样

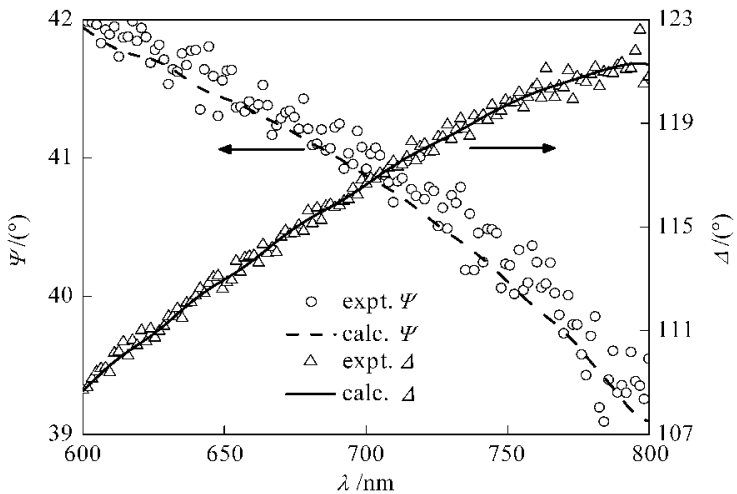

图 4 在 $0.6 \mathrm{~s}$ 时的椭圆偏振光谱实验曲线和由 3 层膜模型 计算得到的理论曲线的比较

Fig.4 Comparison of the experimental ellipsometric spectra at $0.6 \mathrm{~s}$ with the theoretic spectra generated with the three-layer optical model
模型, 也可以很好地拟合对应于通电后 $2.2 \mathrm{~s}$ (图 1 中 标记为 $\mathrm{B}$ 点)的第 2 次原位栯圆偏振光谱数据. 比较 发现, 阻挡层厚度随时间的延长而增大, 这与电流随 时间减小的实验事实相符.

随着反应的进行, $\mathrm{Al}_{2} \mathrm{O}_{3}$ 层靠近溶液一侧部分开 始发生如下的化学溶解反应 ${ }^{[17]}$ :

$\mathrm{Al}_{2} \mathrm{O}_{3}+6 \mathrm{H}^{+} \rightarrow 2 \mathrm{Al}^{3+}+3 \mathrm{H}_{2} \mathrm{O}$

溶解将形成孔洞, 此即 “孔洞萌生”阶段 ${ }^{[19-20]}$. 采 用如图 5 所示的 “溶液-多孔层 $-\mathrm{Al}_{2} \mathrm{O}_{3}$ 层- $\mathrm{Al}$ 基底” 4 层膜光学模型可以比前述 3 层膜模型更好地拟合对 应于通电后 $3.5 \mathrm{~s}$ (图 1 中标记为 $\mathrm{C}$ 点)的第 3 次原位椭 圆偏振光谱数据, 其中多孔层可看作由 $\mathrm{Al}_{2} \mathrm{O}_{3}$ 和溶 液按照一定比例组成, 其等效复折射率 $n$ 可以按照 有效介质近似(EMA)计算而得 ${ }^{[27-28]}$ :

$$
\frac{n^{2}-1}{n^{2}+2}=\phi_{\text {solution }} \frac{n_{\text {solution }}^{2}-1}{n_{\text {solution }}^{2}+2}+\left(1-\phi_{\text {solution }}\right) \frac{n_{\mathrm{Al}_{2} \mathrm{O}_{3}}^{2}-1}{n_{\mathrm{Al}_{2} \mathrm{O}_{3}}^{2}+2}
$$

式中 $n_{\text {solution }}$ 和 $n_{\mathrm{Al}_{2} \mathrm{O}_{3}}$ 分别表示 $\mathrm{H}_{2} \mathrm{SO}_{4}$ 溶液和 $\mathrm{Al}_{2} \mathrm{O}_{3}$ 的 复折射率, $\phi_{\text {solution }}$ 表示溶液在多孔层中的体积分数. 由于溶液填充孔洞, 因此可以认为溶液的体积分数 等于多孔层中孔洞的体积分数, 即孔隙率, 它与孔洞 的密度、直径有关. 值得说明的是, 尽管在上述 4 层 膜光学模型中, 我们同时拟合 $\mathrm{Al}_{2} \mathrm{O}_{3}$ 层厚度 $\left(d_{\mathrm{Al}_{2} \mathrm{O}_{3}}\right)$ 、 多孔层的厚度 $\left(d_{\mathrm{PL}}\right)$ 及其中溶液的体积分数 $\left(\phi_{\text {solution }}\right)$, 但并不能很好地拟合对应于图 1 中 $A 、 B$ 点的原位 椭圆偏振光谱数据, 意味着在 $\mathrm{B}$ 点对应时刻 $(2.2 \mathrm{~s})$ 前还没有孔洞萌生. 由于刚萌生的孔洞非常细小, 很 难应用常规方法进行研究, 通常是和仅生成阻挡型
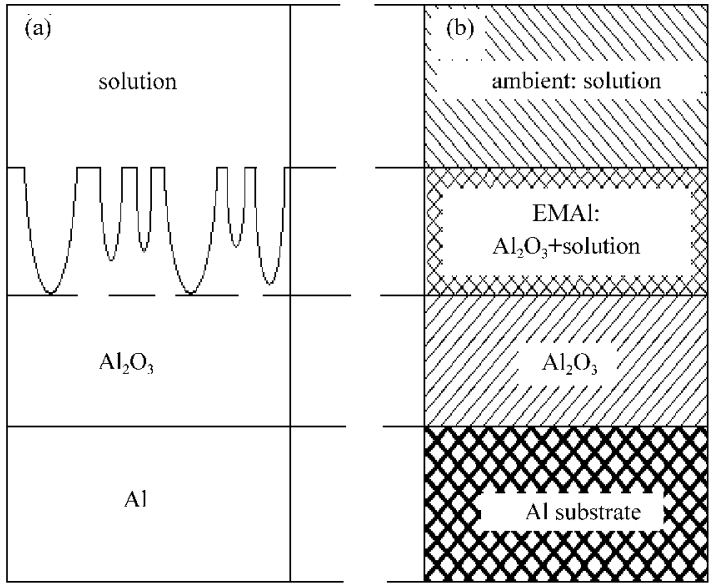

图 5 解析孔洞萌生阶段椭圆偏振光谱实验数据的“ $\mathrm{H}_{2} \mathbf{S O}_{4}$ 溶液-多孔层- $\mathrm{Al}_{2} \mathrm{O}_{3}$ 层-Al 基底”4 层膜模型

Fig.5 Four-layer model of " $\mathrm{H}_{2} \mathrm{SO}_{4}$ solution-porous layer- $\mathrm{Al}_{2} \mathrm{O}_{3}-\mathrm{Al}$ substrate"used to fit the ellipsometric data

(a) physical model; (b) optical model 


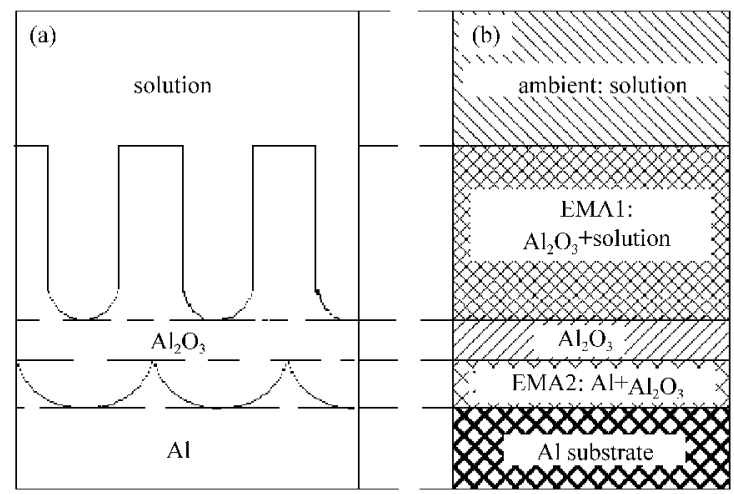

图 6 解析孔洞生长阶段椭圆偏振光谱实验数据的“ $\mathrm{H}_{2} \mathrm{SO}_{4}$ 溶液-多孔层- $\mathrm{Al}_{2} \mathrm{O}_{3}$ 层-界面层-Al 基底”5 层膜模型

Fig.6 Five-layer model of " $\mathrm{H}_{2} \mathrm{SO}_{4}$ solution-porous layer- $\mathrm{Al}_{2} \mathrm{O}_{3}$-interphase layer-Al substrate" used to fit the ellipsometric data

(a) physical model; (b) optical model

氧化铝膜的电流-时间曲线相比较以确定孔洞的萌 生时间: 当仅生成阻挡型氧化铝膜时, 电流单调减 小; 而生成多孔型氧化铝膜时电流会出现先减小再 增大最后逐渐稳定的过程, 故往往将电流出现差别 的时刻作为孔洞萌生的开始 ${ }^{[2]}$, 也有文献 ${ }^{[29]}$ 认为, 孔 洞萌生于电流-时间曲线上最低点对应时间的 $1 / 2$. 然而, 由于阻挡型和多孔型膜在不同电解质、电压等 条件下生成, 因此上述方法并不准确. 我们高灵敏度 的原位椭圆偏振光谱实验为辨别孔洞萌生提供了实 验依据, 结果表明孔洞的产生非常接近于电流-时 间曲线上的最低点.

然而, 前述的 4 层膜模型不能拟合对应于通电 后 $5.0 \mathrm{~s}$ (图 1 中标记为 $\mathrm{D}$ 点)的第 4 次原位椭圆偏振 光谱数据. 考虑到随反应进行, $\mathrm{Al}_{2} \mathrm{O}_{3}$ 阻挡层和 $\mathrm{Al}$ 基 底界面逐渐呈球弧状而出现由 $\mathrm{Al}_{2} \mathrm{O}_{3}$ 阻挡层和 $\mathrm{Al}$ 基底组成的 “界面层”, 光学模型演变为如图6所示的 “溶液-多孔层 $-\mathrm{Al}_{2} \mathrm{O}_{3}$ 层-界面层- $\mathrm{Al}$ 基底” 5 层膜模 型 ${ }^{[15-16]}$, 其中界面层的等效光学常数可以由 $\mathrm{Al}_{2} \mathrm{O}_{3}$ 和 $\mathrm{Al}$ 的光学常数按有效介质近似计算而得. 用此 5 层 膜模型, 同时拟合多孔层的厚度及其中溶液的体积 分数、 $\mathrm{Al}_{2} \mathrm{O}_{3}$ 层厚度、界面层厚度 $\left(d_{\mathrm{IL}}\right)$ 及其中 $\mathrm{Al}_{2} \mathrm{O}_{3}$ 的 体积分数, 可以很好地解析剩余所有 60 次椭圆偏振 光谱数据.

所有拟合结果如图7(a-e)所示. 图中字母 B、C、 E对应于图1中相应字母表示的时刻. 由图7 $(a, b)$ 可 以发现, $26.2 \mathrm{~s}$ (图1中标记为 $\mathrm{E}$ 点)之后, 多孔层孔隙 率 $\phi_{\text {solution }}$ (等同于溶液的体积分数) 几乎不变, 而仅仅 厚度 $d_{\mathrm{PL}}$ 随时间改变, 表明此时体系进人“多孔层稳

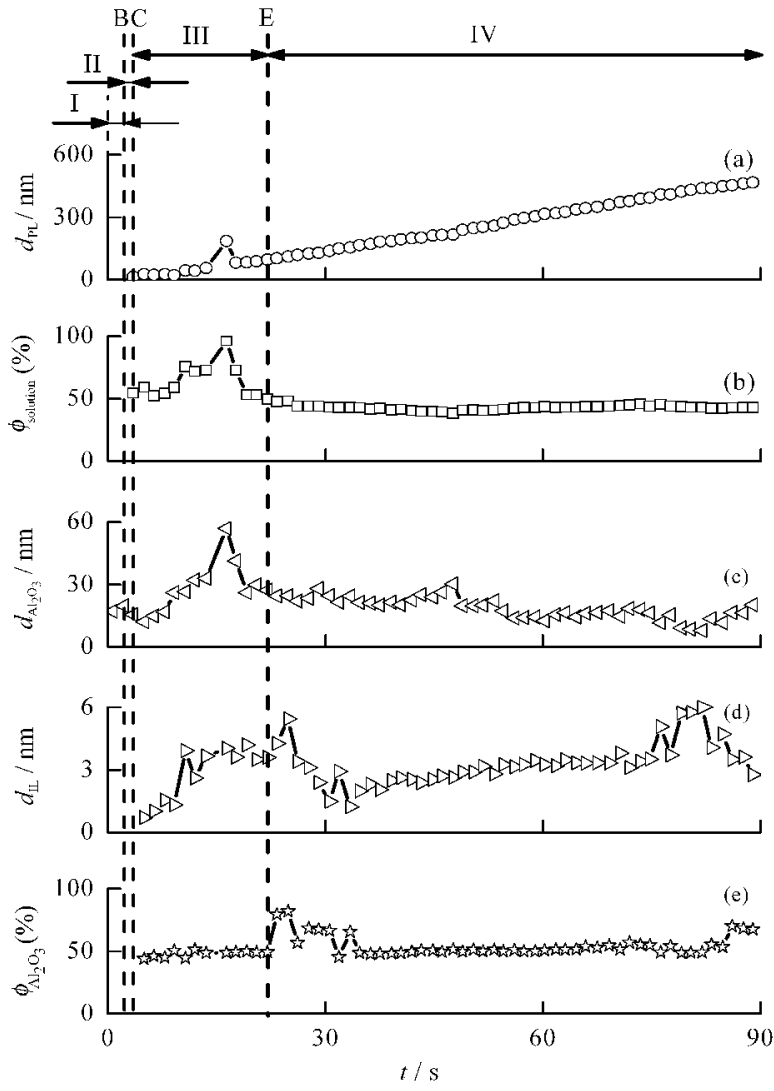

图 7 原位椭圆光谱拟合结果

Fig.7 Results of deconvoluting of the in situ ellipsometric spectra

I, II, III, and IV correspond to the stages of the barrier layer grows, pores form, pores enlarge, and pores grow at a stable rate, respectively. The lines B, C, and E correspond to the time of $2.2,3.5$, and $26.2 \mathrm{~s}$, which are marked as the same letters in Fig.l.

$\phi_{\mathrm{A}_{2} \mathrm{O}_{3}}$ : volume fraction of $\mathrm{Al}_{2} \mathrm{O}_{3} ; \phi_{\text {solution: }}$ : volume fraction of solution;

$d_{\mathrm{LL}}$ : interface layer thickness; $d_{\mathrm{PL}}$ : porous layer thickness

定生长”阶段. 文献中通常以电流达到稳定(本实验 中 $21.0 \mathrm{~s}$ ) 作为此阶段的标志 ${ }^{[29]}$, 但原位椭圆偏振光 谱实验结果表明多孔层稳定生长应稍晚于电流稳 定. 在孔洞萌生与多孔层稳定生长阶段之间, 则是 “孔洞发展” 阶段. 此阶段由原位椭圆偏振光谱计算 得到的各膜层厚度、组成改变的规律不明显, 这可能 与旧孔洞发展的同时有新孔洞萌生(即孔洞萌生与 发展阶段重叠) ${ }^{[20]}$ 以及孔洞同时横向、纵向发展 ${ }^{[30]}$ 有 关.

综上所述, 根据解析椭圆偏振光谱实验数据的 光学模型及计算结果, 可以清楚地分辨铝阳极氧化 过程中阻挡层形成、孔洞萌生、孔洞发展以及多孔层 稳定生长等阶段(在图7中分别以I、II、III、IV表示). 文 献中通常采用非原位测量方法对上述 4 个阶段进行 研究 ${ }^{[1,213,30-31]}$, 由于其测量的不连续性而可能遗漏各 
阶段演变的部分信息. 本文采用高时间分辨率、高灵 敏度的原位椭圆偏振光谱电化学方法详细地获得了 各阶段的界面层、阻挡层以及多孔层的组成、厚度变 化的动态信息, 为各阶段的划分提供了实验依据.

进一步研究发现, 可以用线性关系拟合“多孔层 稳定生长”阶段多孔层厚度随时间 $t$ 的改变, 其方程 及相关系数 $R^{2}$ 为:

$d_{\mathrm{PL}}=5.8 t-40.2, R^{2}=0.997$

上式表明多孔层的生长速度约为 $5.8 \mathrm{~nm} \cdot \mathrm{s}^{-1}$, 由 此可估算制备一定厚度的多孔阳极氧化铝纳米模板 所需要的时间. 此生长速度与文献报道相近条件(21 ${ }^{\circ} \mathrm{C}, 3 \mathrm{~mol} \cdot \mathrm{L}^{-1} \mathrm{H}_{2} \mathrm{SO}_{4}$ 溶液中, $10 \mathrm{~mA} \cdot \mathrm{cm}^{-2}$ 恒电流阳极 氧化)下采用非原位方法测定的多孔层生长速度 5.9 $\mathrm{nm} \cdot \mathrm{s}^{-1}$ 非常接近 ${ }^{[16}$. 另外, 通常认为阻挡层厚度 $d_{\mathrm{Al}_{2} \mathrm{O}_{3}}$ 与制备电压的关系为 $1.0 \mathrm{~nm} \cdot \mathrm{V}^{-1}[24]$, 对应于本实验 $16.0 \mathrm{~V}$ 电压的阻挡层厚度为 $16.0 \mathrm{~nm}$, 此数值与根据 椭圆偏振光谱实验计算得到的多孔层稳定生长阶段 的阻挡层厚度平均值 $18.2 \mathrm{~nm}$ 非常接近. 上述结果 表明我们的模型和计算结果的合理性.

\section{3 结 论}

借助于合理的理论模型, 原位椭圆偏振光谱法 可以很好地用于研究组成、厚度同时改变的多层膜 复杂体系, 为深人理解电化学反应机制提供更丰富 的信息. 本文利用高时间分辨率、高灵敏度的原位椭 圆偏振光谱法研究了铝阳极氧化过程, 结果表明: (1) 利用恰当的光学模型以及有效介质近似可以很 好地解析铝阳极氧化过程中的原位椭圆偏振光谱实 验数据, 从而获得界面层、阻挡层以及多孔层的组成、 厚度变化的动态信息; 根据所用光学模型及计算结 果, 可清楚地分辨铝阳极氧化过程中的各个阶段; (2) 多孔层稳定生长阶段中, 阻挡层厚度及多孔层 的孔隙率几乎不变而多孔层厚度随时间线性增长, 其速度为 $5.8 \mathrm{~nm} \cdot \mathrm{s}^{-1}$.

\section{References}

1 Chik, H.; Xu, J. M. Mater. Sci. Eng. R, 2004, 434: 103

2 Losic, D.; Lillo, M.; Losic, J. D. Small, 2009, 5: 1392

3 Rao, C. N. R.; Govindaraj, A. Adv. Mater., 2009, 21: 4208

4 Tao, F. F.; Xu, Z. Acta Phys. -Chim. Sin., 2009, 25: 977 [陶菲菲, 徐 正. 物理化学学报, 2009, 25: 977]

5 Chen, W.; Wu, J. S.; Xia, X. H. ACS nano, 2008, 2: 959

6 Chen, W.; Wang, F. B.; Ruan, M. H.; Xu, J. J.; Xia, X. H. Sci. Adv.
Mater., 2009, 1: 25

7 Chen, W.; Wu, J. S.; Yuan, J. H.; Xia, X. H.; Lin, X. H. J. Electroanal. Chem., 2007, 600: 257

8 Lee, W.; Ji, R.; Goesele, U.; Nielsch, K. Nat. Mater., 2006, 5: 741

9 Chen, S. S.; Ling, Z. Y.; Hu, X.; Yang, H.; Li, Y. J. Mater. Chem., 2010, 20: 1794

10 Sabzi, R.; Kant, K.; Losic, D. Electrochemica Acta, 2010, 55: 1829

11 Gong, Y. L.; Wang, W.; Wang, H.; Guo, H. T. Acta Phys. -Chim. Sin., 2004, 20: 199 [巩运兰, 王 为, 王 惠, 郭鹤桐. 物理化 学学报, 2004, 20: 199]

12 Stanton, L. G.; Golovin, A. A. Phys. Rev. B, 2009, 79: 035414

13 Azzam, R. M. A.; Bashara, N. M. Ellipsometry and polarized light. Amsterdam: North-Holland Publishing Company, 1977: 283, 452

14 Lei, J. L.; Li, L. J.; Cai, S. M.; Zhang, S. T.; Li, D.; Yang, M. Z. Acta Phys. -Chim. Sin., 2001, 17: 1107 [雷惊雷, 李凌杰, 蔡生 民, 张胜涛, 李 荻, 杨迈之. 物理化学学报, 2001, 17: 1107]

15 Laet, J. D.; Vanhellemont, J.; Terryn, H.; Vereccken, J. Appl. Phys. A, 1992, 54: 72

16 Schnyder, B.; Kötz, R. J. Electroanal. Chem., 1992, 339: 167

17 Laet, J. D.; Terryn, H.; Vereccken, J. Thin Solid Films, 1998, 320: 241

18 Kooij, E. S.; Wormeester, H.; Gâlcă, A. C.; Poelsema, B. Electrochem. Solid-State Lett., 2003, 6: B52

19 Parkhutik, V. P.; Shershulsky, V. I. J. Phys. D-Appl. Phys., 1992, 25: 1258

20 Zhu, Z. F. Techniques of anodic oxidizing and surface treating for aluminum alloys. Beijing: Chemical Industry Press, 2004: 94 [朱祖芳. 铝合金阳极氧化与表面处理技术. 北京: 化学工业出版 社, 2004: 94]

21 Wu, Z.; Richter, C.; Menon, L. J. Electrochem. Soc., 2007, 154: E8

22 Okabe, H.; Hayakawa, M.; Matoba, J.; Naito, H.; Oka, K. Rev. Sci. Instrum., 2009, 80: 083104

23 Palik, E. D. Handbook of Optical Constants of Solids (III). San Diego: Academic Press, 1998: 653

24 Hoar, T. P.; Yahalom, J. J. Electrochem. Soc., 1963, 110: 614

25 Chen, T. X. Experimental electrochemistry. Xiamen: Xiamen University Press, 1993: 226 [陈体衔. 实验电化学. 厦门: 厦门大 学出版社, 1993: 226]

26 Diggle, J. W.; Downie, T. C.; Goulding, C. W. Chem. Rev., 1969, 69: 365

27 Aspnes, D. E.; Kinsbron, E.; Bacon, D. D. Phys. Rev. B, 1980, 21: 3290

28 Fujiwara, H. Spectroscopic dllipsometry, principles and applications. West Sussex: John Willy \& Sons Ltd., 2007: 177

29 Siejka, J.; Ortega, C. J. Electrochem. Soc., 1977, 124: 883

30 Xu, Y.; Thompson, G. E.; Wood, G. C. J. Chin. Soc. Corr. Prot., 1989, 9: 1 [徐 源, Thompson, G. E., Wood, G. C. 中国腐蚀与 防护学报, 1989, 9: 1]

31 Thompson, G. E.; Furneaux, R. C.; Wood, G. C.; Richardson, J. A.; Goode, J. S. Nature, 1978, 272: 433 